\title{
Giant ferroelectric polarization and electric reversal of strong spontaneous magnetization in multiferroic $\mathrm{Bi}_{2} \mathrm{FeMoO}_{6}$
}

\author{
Peng Chen ${ }^{1}$ and Bang-Gui Liu ${ }^{1,2 \text {, }, ~}$ \\ ${ }^{1}$ Beijing National Laboratory for Condensed Matter Physics, \\ Institute of Physics, Chinese Academy of Sciences, Beijing 100190, China \\ ${ }^{2}$ School of Physical Sciences, University of Chinese Academy of Sciences, Beijing 100190, China
}

(Dated: March 30, 2021)

\begin{abstract}
$\mathrm{BiFeO}_{3}$ is the most famous multiferroic material, but it has no strong spontaneous magnetization due to its antiferromagnetism. Here we show that giant ferroelectric polarization and strong spontaneous magnetization can be both realized in double perovskite $\mathrm{Bi}_{2} \mathrm{FeMoO}_{6}$ with $\mathrm{R} 3$ (\#146) space group based on $\mathrm{BiFeO}_{3}$. Our first-principles phonon spectra establishes that this multiferroic R3 phase is stable. Our systematic calculations show that it is a spin-polarized semiconductor with gap reaching to $0.54 \mathrm{eV}$ and has a strong ferroelectric polarization of $85 \mu \mathrm{C} / \mathrm{cm}^{2}$. This ferroelctricity is comparable with that of $\mathrm{BiFeO}_{3}$, but here obtained is a strong ferrimagnetism with net magnetic moment of $2 \mu_{B}$ per formula unit and Curie temperature of $650 \mathrm{~K}$. Both ferroelectric polarization and magnetic easy axis are shown to be in pseudocubic [111] orientation. Our further analysis shows that the macroscopic spontaneous magnetization can be deterministically reversed through a three-step path by external electric field. Therefore, we believe that this $\mathrm{Bi}_{2} \mathrm{FeMoO}_{6}$ material can be used to design new multifunctional materials and achieve high-performance devices.
\end{abstract}

\section{INTRODUCTION}

One of the most significant advantages of magnetoelectric (ME) multiferroics is that the ME coupling ${ }^{1,2}$ can be used to manipulate magnetic properties by external electric fields, or to control electric properties by external magnetic field ${ }^{3}$. However, one of challenges is to find promising multiferroic materials that have both large magnetization and enormous ferroelectric polarization beyond room temperature. So far, the perovskite $\mathrm{BiFeO}_{3}$ (BFO) is the only such single-phase multiferroic material ${ }^{4-12}$. Both bulk and thin film BFO samples can exhibit strong ferroelectricity $\left(P \simeq 100 \mu \mathrm{C} / \mathrm{cm}^{2}\right)$ with high Curie temperature $1100 \mathrm{~K}^{12}$ and strong antiferromagnetism with magnetic Neel temperature $650 \mathrm{~K}^{13-16}$. The Dzyaloshinskii-Moriya (DM) ${ }^{17,18}$ interaction can induce in it a weak ferromagnetism with a remanent magnetization of $0.02 \mu_{B} / \mathrm{Fe}^{19,20}$. It has been revealed theoretically and experimentally that $\mathrm{BiFeO}_{3}$-based systems can display novel coupling phenomenon between spontaneous electric polarization in the pseudocubic [111] direction and the weak ferromagnetism perpendicular to the [111] direction ${ }^{20,21}$, which makes it possible to control the weak ferromagnetism through the electric polarization switching by an external electric field.

A strong macroscopic magnetization above room temperature is highly desirable for energy-efficient spintronic devices 3,8 . Here, we show giant ferroelectric polarization and electric reversal of strong macroscopic spontaneous magnetization in multiferroic $\mathrm{Bi}_{2} \mathrm{FeMoO}_{6}$ (BFMO) phase with R3 space group. Its high-temperature ferrimagnetism have been proposed ${ }^{22}$ and its multiferroic phase (with a small ferroelectric polarization and a magnetic Curie temperature beyond room temperature) has been realized experimentally ${ }^{23}$. Our first-principles calculations reveal that it is a semiconductor with gap reaching to $0.54 \mathrm{eV}$, and has ferroelectric polarization of $85 \mu \mathrm{C} / \mathrm{cm}^{2}$ and strong spontaneous ferrimagnetism with net magnetic moment of $2 \mu_{B}$ per formula unit. Considering its ferroelectricity is similar to that of the $\mathrm{BiFeO}_{3}$, we believe that its ferroelectric polarization can persist beyond room temperature as its macroscopic spontaneous magnetization does. Furthermore, our study shows that its magnetic easy axis is collinear with its ferroelectric polarization vector in the pseudocubic [111] directions, and its spontaneous magnetization can be deterministically reversed through three switching steps of its ferroelectric polarization under an applied electric field. More detailed results will be presented in the following.

\section{COMPUTATIONAL METHODS}

We use projector augmented-wave (PAW) ${ }^{24}$ plus pseudo-potential methods within the density functional theory ${ }^{25,26}$, as implemented in the Vienna ab initio simulation package (VASP) ${ }^{27,28}$, to optimize the crystal structures and then study the electronic structures, ferroelectricity, and magnetic properties. The spinpolarized generalized-gradient approximation (GGA) $\stackrel{29}{ }$, to the electronic exchange-correlation functional, is used to do structure optimization. To make the structures compatible with different magnetic configurations and atomic distortions, calculations were performed using a 40-atom super-cell which can be considered as doubling the ideal perovskite structure along the three Cartesian directions. Plane wave basis set with a maximum kinetic energy of $500 \mathrm{eV}$ and $3 \times 3 \times 3 \mathrm{k}$-point mesh generated with the Monkhorst-Pack scheme ${ }^{30}$ were used. During optimization, all of the structures were fully relaxed until the largest force between the atoms become less than $1 \mathrm{meV} / \AA$. When we obtain very small total energy difference between two structures in terms of the pseudo- 
potential method, we use the full-potential method to more accurately describe the total energy difference in order to improve the total energy comparison 31 .

Phonon spectra are calculated with first-principles perturbation method with norm-conserving pseudopotentials as implemented in package QuantumESPRESSO ${ }^{32}$. The magnetic anisotropy energy (MAE) due to the spin-orbit coupling is determined by the force theorem, and calculated in terms of the total energy with respect to polar and azimuthal angle along the [111] direction. Switching paths were determined using the 'Nudged Elastic Band' (NEB) ${ }^{33}$ method which can give the most energetically favourable intermediate configuration between the initial and final states. We use the modified Becke-Johnson exchange potential to accurately calculate semiconductor gaps 31,34 . We use modern Berry phase method ${ }^{35}$ and take both GGA $+\mathrm{U}^{36,37}$ and HSE06 $\underline{38,39}$ schemes to calculate electric polarization.

\section{RESULTS AND DISCUSSIONS}

\section{A. Optimized structure}

Possible crystalline and magnetic structures. Double perovskite $\mathrm{Bi}_{2} \mathrm{FeMoO}_{6}$ (BFMO) can be related with the famous perovskite $\mathrm{BiFeO}_{3}$ (BFO) by considering that $\mathrm{BFMO}$ is obtained through partially substituting Fe in BFO by Mo. In order to seek stable phases of BFMO, we start with 12 basic space groups of $\mathrm{Bi}_{2} \mathrm{FeMoO}_{6}$ allowed by group analysis ${ }^{40}-44$, and then we optimize the crystal structures and calculate the total energies by considering three ferroelectric distortions and four magnetic configurations: one ferromagnetic (FM) and three antiferromagnetic (AFM) or ferrimagnetic (FIM) orders of A-type, C-type, and G-type. Actually, all possible structures related with the 12 space groups have been considered, including the different magnetic structures and possible ferroelectric distortions of Bi atoms in three cartesian directions. These cover cubic, tetrahedral, orthorhombic, rhombohedral, hexagonal, and monoclinic crystal systems. The most stable six phases are summarized in Table [I where we present their space groups, Glazor notes for oxygen-octahedron tilting, volumes, net magnetic moments, and total energies (with the lowest energy set to zero). It is interesting and reasonable that the most stable magnetic configuration is the FIM order of G-type for all the six structures shown in Table I. Our results also show that the R3 phase is semiconductive, the $\mathrm{P} 2_{1} / n$ and $\mathrm{R} \overline{3}$ are half-metallic, and the other three are normally metallic.

The R3 phase as a multiferroic material. Our total energy comparison reveals that the R3 is the ground state phase with the lowest energy, and it exhibits ferroelectric property. There exists a $\mathrm{P} 2_{1} / n$ metastable magnetic phase which is higher by only $75 \mathrm{meV}$ per formula unit than the ground state phase. This R3 phase can be considered to be distorted from the $\mathrm{R} \overline{3}$ structure, and is
TABLE I. Space groups, Glazor notes, volumes ( $V$, in $\AA^{3} /$ f.u. ), magnetic moments $\left(M\right.$, in $\mu_{B} /$ f.u. $)$, and total energies $(\Delta E$, in $\mathrm{meV} /$ f.u. $)$ of the lowest six of the structures.

\begin{tabular}{cccccc}
\hline \hline No. & Space group & Glazor & $V$ & $M$ & $\Delta E$ \\
\hline 1 & $\mathrm{R} 3$ & $a^{-} a^{-} a^{-}$ & 133.27 & 2 & 0 \\
2 & $\mathrm{P} 21 / n$ & $a^{-} a^{-} c^{+}$ & 128.62 & 2 & 75. \\
3 & $\mathrm{R} \overline{3}$ & $a^{-} a^{-} a^{-}$ & 131.94 & 2 & 401. \\
4 & $\mathrm{C} 2 / \mathrm{m}$ & $a^{0} b^{-} b^{-}$ & 129.15 & 2.5 & 364. \\
5 & $\mathrm{I} 4 / \mathrm{m}$ & $a^{0} a^{0} c^{-}$ & 127.93 & 2.3 & 763. \\
6 & $\operatorname{Pn} \overline{3}$ & $a^{+} a^{+} a^{+}$ & 128.89 & 2.2 & 921. \\
\hline \hline
\end{tabular}

comparable with the ground state R3c bulk phase in the case of BFO. Here, we focus on the R3 phase because it has strong ferrimagnetic order, large ferroelectric polarization, and useful magneto-electric coupling, as will be shown in the following. We illustrate the crystal structure, local octahedral tilting, and crystal deformation in Fig 1 The O-Fe-O, O-Mo-O, and Fe-O-Mo bond angles are reduced to $163.3^{\circ}, 168.6^{\circ}$, and $150.0^{\circ}$, respectively, which reflect substantial crystal deformation. We have calculated the phonon spectra of the $\mathrm{P} 21 / n$ and $\mathrm{R} 3$ phases and present that of the R3 structure in Fig. 2(a). These results show that there is not any instability in these two cases. Because the other phases are at least 75 $\mathrm{meV} /$ f.u. higher than the rhombohedral R3 phase, we believe that this R3 phase can be synthesized as epitaxial (111) thin films on appropriate substrates, like those of $\mathrm{BFO}^{45-48}$.

\section{B. Electronic structure and magnetism}

Because GGA usually underestimate semiconductor gaps, we use the modified Becke-Johnson (mBJ) exchange functional to study the electronic structure. Presented in Fig 2(b) is our mBJ calculated spin-dependent density of states (DOS) of the optimized R3 structure of $\mathrm{Bi}_{2} \mathrm{FeMoO}_{6}$. Our mBJ calculated gap, $0.54 \mathrm{eV}$, should be a much better value for the true gap because mBJ has been proved to give accurate semiconductor gaps for most of semiconductors and, more importantly, our mBJ gap for $\mathrm{BFO}$ is equivalent to $2.1 \mathrm{eV}$ which is in good agreement with experimental results of $2.5 \mathrm{eV}^{\underline{49}, 50}$. Our analysis reveals that in the spin-up DOS, the three filled $\mathrm{Fe}$ t2 $\mathrm{g}$ bands merges into the oxygen $\mathrm{p}$ bands and the two filled Fe eg bands are separated from them, and the lowest empty bands, mainly from Mo $4 \mathrm{~d}$ states, are above $1.75 \mathrm{eV}$. It can be seen that the semiconductor gap, 0.54 $\mathrm{eV}$, is formed between the three filled Mo $4 \mathrm{~d}$ bands and the three empty Fe 3d ones in the spin-down channel. Totally, we have five Fe d electrons in the spin-up channel and three Mo d electrons in the spin-down channel, and thus the magnetic moment per formula unit is equivalent to $2 \mu_{B}$, which is consistent with the calculated data. We also use other exchange-correlation schemes to calculate the semiconductor gap and thereby make 

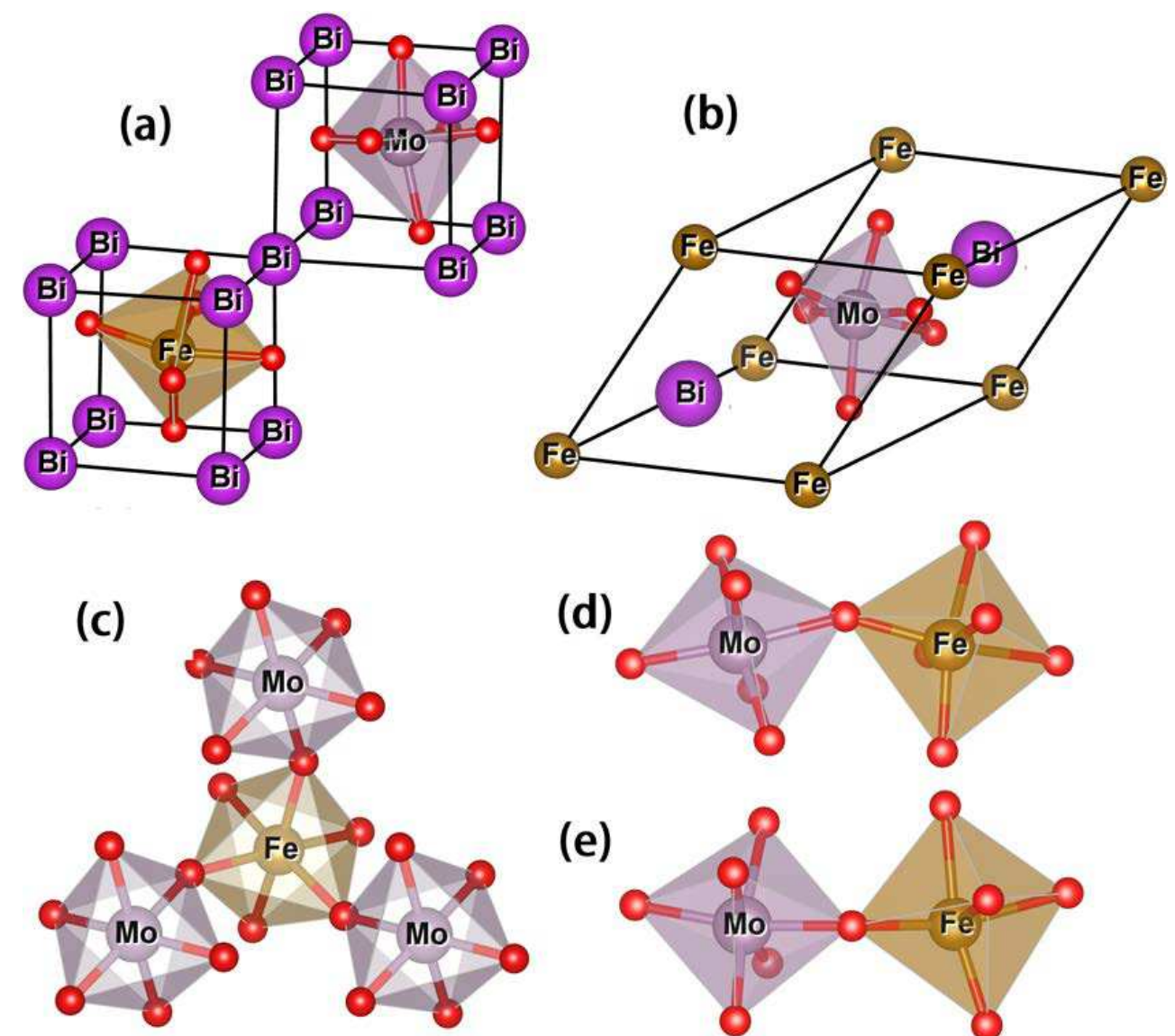

(d)

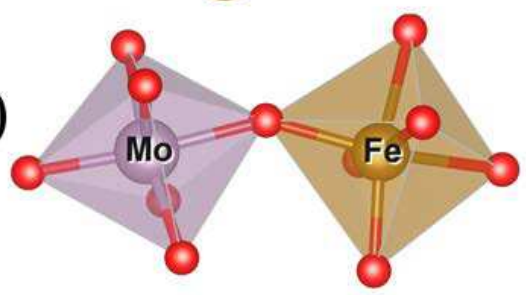

(e)

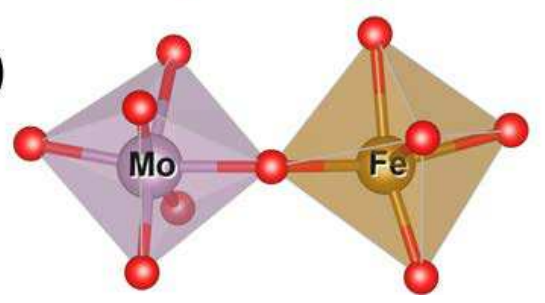

FIG. 1. The key structural features: (a) the crystal structure of BFMO showing the alternate occupation of Fe and Mo at the B-sites along the [111] direction, (b) the primitive cell of BFMO with the O octahedron of Mo shown, (c) the relative rotation projected on the (111) plane of an $\mathrm{O}$ octahedron of Fe with respect to the nearest $\mathrm{O}$ octahedra of Mo, and (d) and (e) the two neighboring $\mathrm{O}$ octahedra of Fe and Mo projected on the plane of the Fe-O-Mo triangle and projected on the perpendicular plane including the Fe and Mo ions.

sure that the semiconductor gap is true for the R3 phase of $\mathrm{Bi}_{2} \mathrm{FeMoO}_{6}$. On the other hand, we also calculate spin exchange energies and Curie temperatures for the R3 phase, confirming the high magnetic Curie temperature of $650 \mathrm{~K}^{22}$.

\section{Excellent multiferroics}

We have studied the effect of the spin-orbit coupling for the R3 phase. We present the calculated magnetocrystalline anisotropy energy (MAE) as functions of the polar and azimuthal angles in Fig. 2 (c) and (d). Here, the polar and azimuthal angles are defined with respect to the pseudo[111] direction. In contrast to the magnetic easy plane for $\mathrm{BFO}^{20,51}$, $\mathrm{BFMO}$ has a magnetic easy axis and its magnetization tends to align along the pseudocubic [111] axis. Actually, the MAE can be well described by a simple expression, $E_{\mathrm{MA}}=1.5 \sin ^{2} \theta$ in $\mathrm{meV}$, where the polar angle $\theta$ takes values from 0 to $\pi$. On the other hand, we have analyze the ionic positions of the R3 phase projected in the [111] direc- tion and show the result in Fig 2 (e). We observe clear cation displacements with respect to their oxygen environment, which are like those in the BFO which exhibits strong ferroelectricity. Furthermore, we quantitatively investigate the ferroelectricity of BFMO through modern DFT-based calculations with both GGA $+\mathrm{U}^{36,37}$ and HSE06 $\stackrel{38,39}{ }$ methods. For the GGA+U scheme, we use one set of parameter $(\mathrm{U}, \mathrm{J})=(2.5 \mathrm{eV}, 0.5 \mathrm{eV})$ for Mo and three sets for Fe: $(\mathrm{U}, \mathrm{J})=(2 \mathrm{eV}, 0.8 \mathrm{eV}),(4 \mathrm{eV}, 0.8 \mathrm{eV})$, and $(8 \mathrm{eV}, 0.8 \mathrm{eV})$. Our calculations give a large spontaneous ferroelectric polarization $85 . \mu \mathrm{C} / \mathrm{cm}^{2}$ in the [111] direction, which is nearly independent of calculational schemes and parameters. In addition to the high magnetic Curie temperature, we expect a high ferroelectric Curie temperature for this ferroelectricity because it should share the similar mechanism of ferroelectricity with BFO. Therefore, BFMO should be an excellent multiferroic material. 
(a)
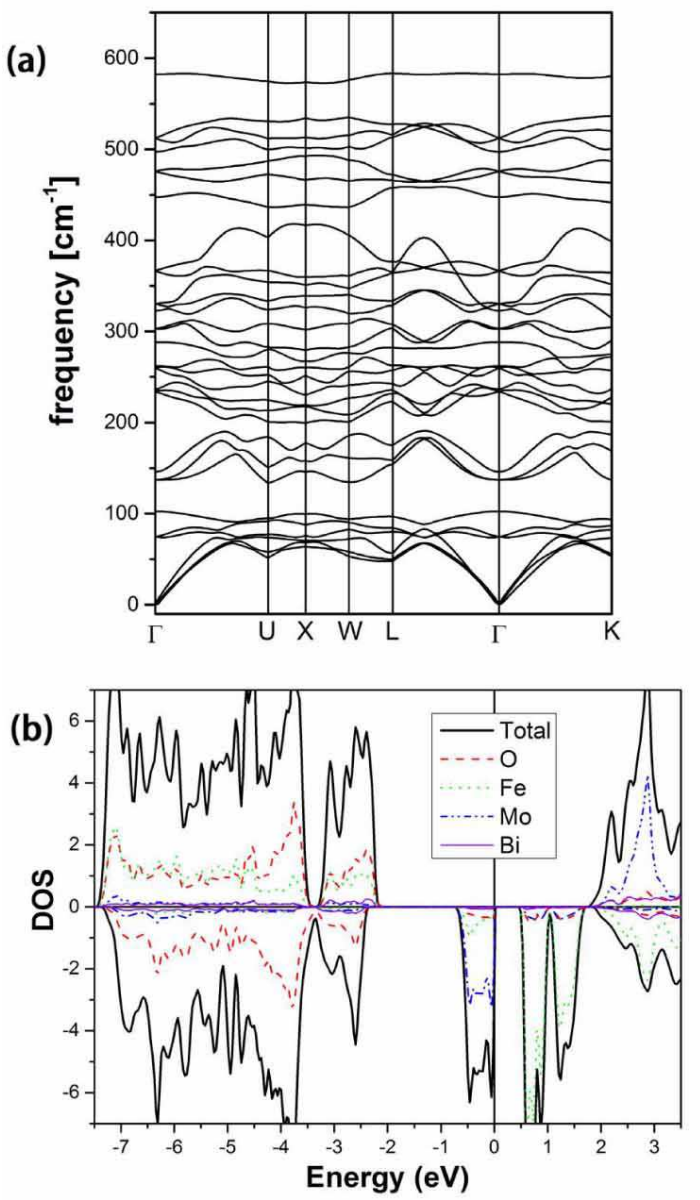

(c)

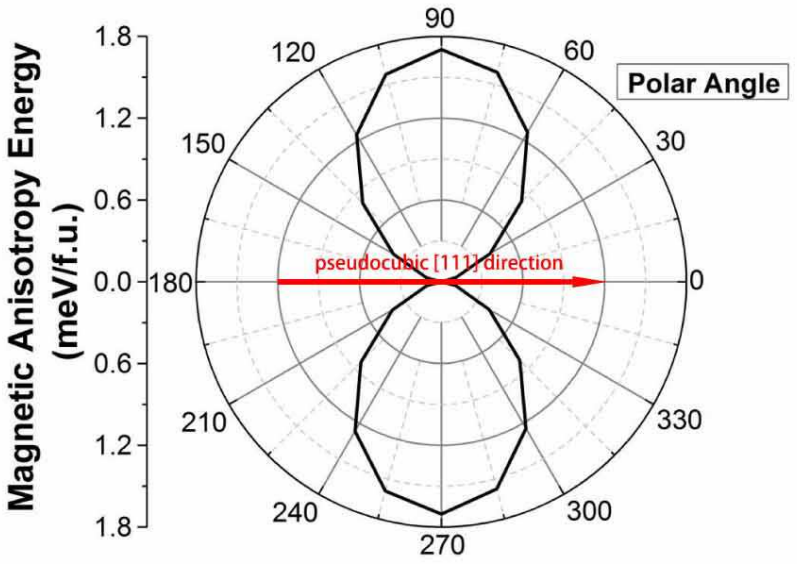

(d)

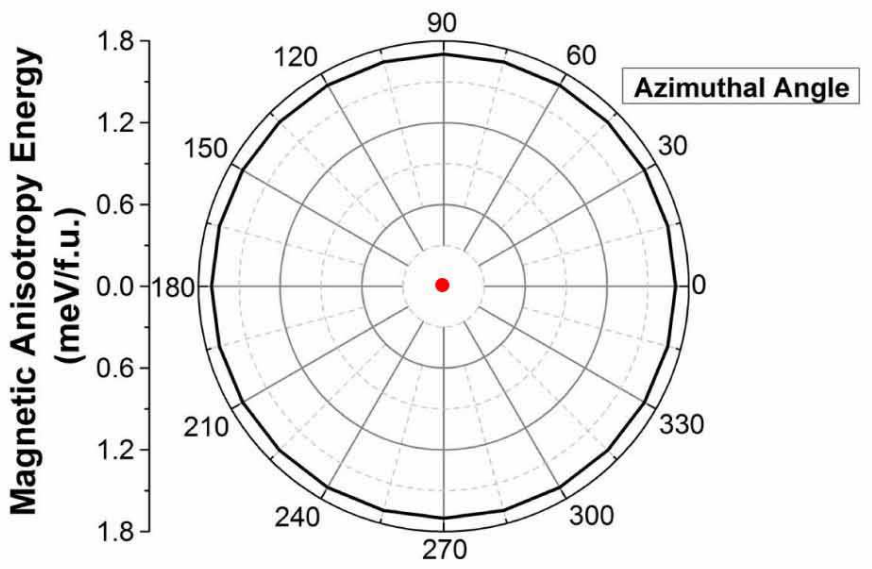

(e)

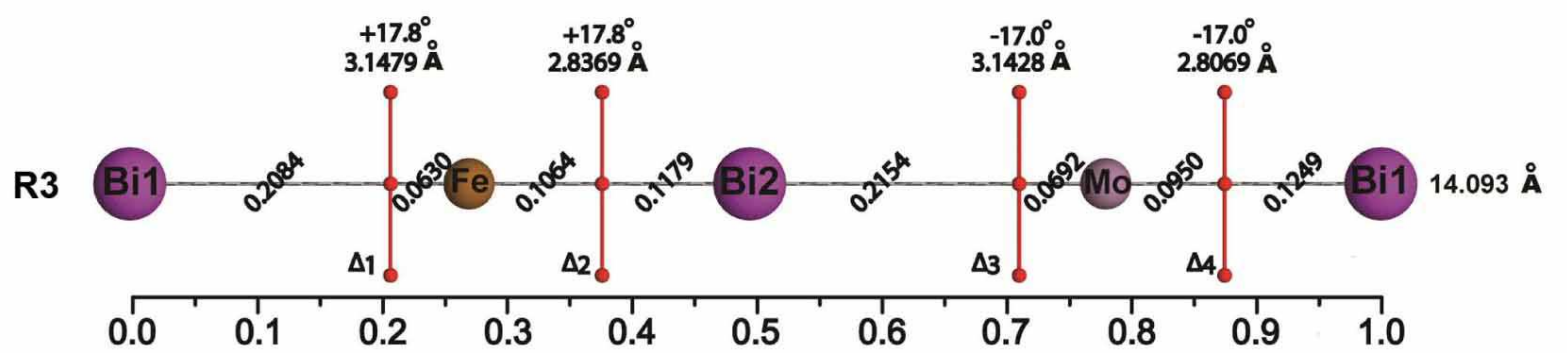

FIG. 2. Basic properties of BFMO: (a) the electronic density of states (DOS), (b) the phonon spectra showing no structural instability, (c) and (d) the relative total energies in a plane including the [111] axis and in the (111) plane, and (e) an analysis of ion displacements. The DOS in (a), in state/eV per primitive cell, shows a net moment $2 \mu_{B}$ and a semiconductor gap 0.54 $\mathrm{eV}$, and the upper half shows spin-up DOS and the lower spin-down DOS. The energies in (c,d) shows a uniaxial easy axis in [111] and an MCA energy of $1.5 \mathrm{meV}$. The skew numbers on the horizontal line in (e) are the relative distances between the positive ions ( $\mathrm{Bi} 1, \mathrm{Fe}, \mathrm{Bi} 2, \mathrm{Mo})$ and the centers of the nearest $\mathrm{O}$ triangles $\left(\Delta_{1}, \Delta_{2}, \Delta_{3}, \Delta_{4}\right)$, and the numbers above the vertical lines show the relative rotational angles around the [111] axis and the edge lengthes of the $\mathrm{O}$ triangles, respectively. There is a $60^{\circ}$ rotation between $\Delta_{1}$ and $\Delta_{2}$, and between $\Delta_{3}$ and $\Delta_{4}$.

\section{Electric reversal of magnetization}

Cubic anisotropy and reversal paths of ferroelectric polarization. Actually, there are eight equivalent [111] directions, which implies that the ferroelectric anisotropy is cubic 21,51 . We can describe the ferroelectric crystalline anisotropy by the following expression,

$$
E_{f}=K\left[1 / 3-\left(\alpha_{1}^{2} \alpha_{2}^{2}+\alpha_{2}^{2} \alpha_{3}^{2}+\alpha_{3}^{2} \alpha_{1}^{2}\right)\right],
$$

where $\left(\alpha_{1}, \alpha_{2}, \alpha_{3}\right)$ denotes the directional cosine of the ferroelectric polarization vector and our calculation gives $K=1.44 \mathrm{eV}$. The ferroelectric polarization anisotropy is plotted in Fig. 3(a), where there are eight minima or bottom points (B0, B1, B2, B3 etc.) and twelve saddle 


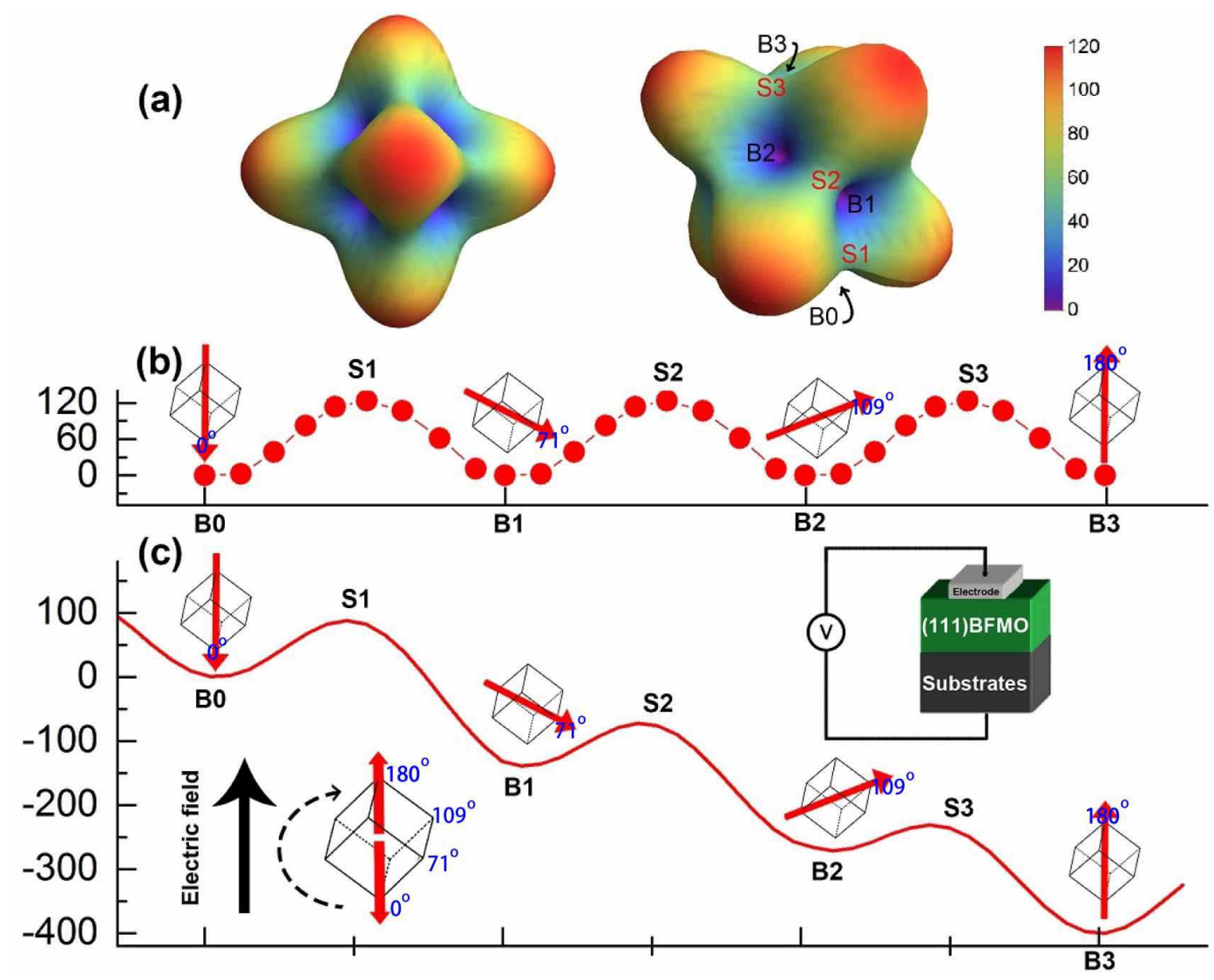

FIG. 3. Reversal of the uniaxial magnetization driven by electric three-step switching of polarization. (a) collinear reversal of polarization through $-P \rightarrow 0 \rightarrow+P$, with energy barriers of $340 \mathrm{meV} /$ f.u.; (b) rotational reversal of polarization through three steps $0^{\circ} \rightarrow 71^{\circ} \hookrightarrow 109^{\circ} \rightarrow 180^{\circ}$, with energy barriers of $120 \mathrm{meV} /$ f.u.; and (c) the three-step reversal of the electric polarization and the uniaxial magnetization with an electric field applied in the $180^{\circ}$ direction. The polar angles accrued at $\mathrm{B} 1, \mathrm{~B} 2$, and $\mathrm{B} 3$ are $71^{\circ}, 109^{\circ}$, and $180^{\circ}$, respectively. Both of the vectors actually rotate by $71^{\circ}$ for each step, with the second step contributing only $38^{\circ}$ to the polar angle.

points (S1, S2, S3 etc). Assuming usual collinear polarization reversal over the $\mathrm{R} \overline{3}$ structure as an intermediate one, $-P \rightarrow 0 \rightarrow+P$, our DFT calculation gives an energy barrier $340 \mathrm{meV}$ per formula unit. In addition, we have found a three-step path for the polarization reversal: $0^{\circ} \rightarrow 71^{\circ} \hookrightarrow 109^{\circ} \rightarrow 180^{\circ}$, where the three nonzero angles describe the polar angles of B1, B2, and B3 with respect to $\mathrm{B} 0$. Along this path, the polarization vector actually changes the equivalent angle of $71^{\circ}$ three times, but the second step is not in the polar angle direction, contributing to only $38^{\circ}$ to the polar angle, as shown in Fig. 3(b). The barrier for each of the three steps, the energy difference between the saddle points and the bottom points, is equivalent to $120 \mathrm{meV}$ per formula unit, substantially lower than that of the collinear reversal. Therefore, this three-step reversal path is favorable in energy.

Electric three-step reversal of strong magnetization. As we have shown above, the magnetic [111] easy axis is collinear with the ferroelectric polarization vector along the [111] direction. The MA energy can be re-written as $E_{\mathrm{MA}}=c_{m e}(\vec{M} \times \vec{P})^{2}$, where $\vec{M}$ is magnetization vec- tor and $c_{m e}(>0)$ describes the magneto-electric coupling strength. As shown in Fig. 3(c), if we apply an electric field antiparallel to the ferroelectric polarization vector, the field can make the energy curve substantially decline. For each of the energy wells, because the left barrier is substantially lower than the right barrier, the polarization vector will try to follow the field. Applying a rotational electric field, from 0 to $180^{\circ}$, can make the polarization vector follow the three-step path for the polarization reversal: $0^{\circ} \rightarrow 71^{\circ} \hookrightarrow 109^{\circ} \rightarrow 180^{\circ}$. Because of $E_{\mathrm{MA}}$, the magnetization tends to stay in the same direction as the ferroelectric polarization vector and will finally reverse, as long as the rotation of the electric field is slow enough.

\section{CONCLUSION}

In conclusion, we have investigated double perovskite $\mathrm{Bi}_{2} \mathrm{FeMoO}_{6}$ through systematic DFT calculations and structural analyses. Our careful GGA optimization reveals that the $\mathrm{Bi}_{2} \mathrm{FeMoO}_{6}$ in the $\mathrm{R} 3$ structure is a ferri- 
magnetic semiconductor and the net moment reaches to $2 \mu_{B}$ per formula unit because the magnetic moments of $\mathrm{Fe}$ and Mo cannot be canceled. The semiconductor gap has been corrected with mBJ potential, reaching to 0.54 eV. Our DFT-based polarization calculations directly shows that the $\mathrm{Bi}_{2} \mathrm{FeMoO}_{6}$ has strong ferroelectric polarization $85 \mu \mathrm{C} / \mathrm{cm}^{2}$ in the pseudocubic [111] direction. Therefore, we believe that the strong ferroelectricity in the $\mathrm{Bi}_{2} \mathrm{FeMoO}_{6}$ can have high Curie temperature far beyond room temperature, because its mechanism should be similar to that of BFO. With the spin-orbit effect into account, our calculation show that the magnetic easy axis is also in the [111] axis. Because the ferroelectric polarization and magnetic easy axis are both in pseudocubic [111] orientation, we show that the strong macroscopic spontaneous magnetization can be deterministically re- versed through a three-step path by external electric field. When realized, the high-quality $\mathrm{Bi}_{2} \mathrm{FeMoO}_{6}$ material, like $\mathrm{BiFeO}_{3}$, can be useful for designing new multifunctional materials and achieve high-performance devices in the future.

\section{ACKNOWLEDGMENTS}

This work is supported by the Nature Science Foundation of China (No. 11574366), by the Strategic Priority Research Program of the Chinese Academy of Sciences (Grant No.XDB07000000), and by the Department of Science and Technology of China (Grant No. 2016YFA0300701).
* bgliu@iphy.ac.cn

1 M. Fiebig, J. Phys. D: Appl. Phys. 38, R123 (2005).

${ }^{2}$ W. Eerenstein, N. D. Mathur and J. F. Scott, Nature 442, 759 (2006).

3 S. Fusil, V. Garcia, A. Barthelemy and M. Bibes, Annu. Rev. Mater. Res. 44, 91 (2014).

4 D. M. Evans, A. Schilling, A. Kumar, D. Sanchez, N. Ortega, M. Arredondo, R. S. Katiyar, J. M. Gregg and J. F. Scott, Nat. Commun. 4, 1534 (2013).

5 A. A. Belik, S. Iikubo, K. Kodama, N. Igawa, S.-I. Shamoto, S. Niitaka, M. Azuma, Y. Shimakawa, M. Takano, F. Izumi and E. Takayama-Muromachi, Chem. Mater. 18, 798 (2006).

6 M.-R. Li, U. Adem, S. R. C. McMitchell, Z. Xu, C. I. Thomas, J. E. Warren, D. V. Giap, H. Niu, X. Wan, R. G. Palgrave, F. Schiffmann, B. Slater, T. L. Burnett, M. G. Cain, A. M. Abakumovi, G. Tendelooi, M. F. Thomas, M. J. Rosseinsky and John B. Claridge, J. Am. Chem. Soc. 134, 3737 (2012).

7 W. Wang, J. Zhao, W. Wang, Z. Gai, N. Balke, M. Chi, H. N. Lee, W. Tian, L. Zhu, X. Cheng, D. J. Keavney, J. Yi, T. Z. Ward, P. C. Snijders, H. M. Christen, W. Wu, J. Shen and X. Xu, Phys. Rev. Lett. 110, 237601 (2013).

8 D. Sando, A. Barthelemy and M. Bibes, J. Phys.: Condens. Matter 26, 473201 (2014).

9 J.-G. Park, M. D. Le, J. Jeong and S. Lee, J. Phys.: Condens. Matter 26, 433202 (2014).

10 G. Catalan and J. F. Scott, Adv. Mater. 21, 2463 (2009).

11 J. Wang, J. B. Neaton, H. Zheng, V. Nagarajan, S. B. Ogale, B. Liu, D. Viehland, V. Vaithyanathan, D. G. Schlom, U. V. Waghmare, N. A. Spaldin, K. M. Rabe, M. Wuttig and R. Ramesh, Science 299, 1719 (2003).

12 J. B. Neaton, C. Ederer, U. V. Waghmare, N. A. Spaldin and K. M. Rabe, Phys. Rev. B 71, 0141131 (2005).

13 J.-M. Moreau, C. Michel, R. Gerson and W. J. James, J. Phys. Chem. Solids 32, 1315 (1971).

14 Y. E. Roginskaya and Y. Tomashpolskii, Zh. Eksp. Teor. Fiz 50, 69 (1966).

15 W. Kaczmarek, M. Polomska and Z. Pajak, Phys. Lett. A 47, 227 (1974).

16 S. Kiselev, G. Zhdanov and R. Ozerov, Dokl. Akad. Nauk SSSR 145, 1255 (1962).
17 I. A. Sergienko and E. Dagotto, Phys. Rev. B 73, 094434 (2006).

18 T. Arima, A. Tokunaga, T. Goto, H. Kimura, Y. Noda and Y. Tokura, Phys. Rev. Lett. 96, 097202 (2006).

19 H. Bea, M. Bibes, S. Petit, J. Kreisel and A. Barthelemy, Philos. Mag. Lett. 87, 165 (2007).

20 C. Ederer and N. A. Spaldin, Phys. Rev. B 71, 060401 (2005).

21 J. T. Heron, J. L. Bosse, Q. He, Y. Gao, M. Trassin, L. Ye, J. D. Clarkson, C. Wang, J. Liu, S. Salahuddin, D. C. Ralph, D. G. Schlom, J. Iniguez, B. D. Huey and R. Ramesh, Nature 516, 370 (2014).

22 S.-D. Li, P. Chen and B.-G. Liu, AIP Advances 3, 012107 (2013).

23 S. Ravi and C. Senthilkumar, Mater. Express 5, 68 (2015).

24 P. E. Blochl, Phys. Rev. B 50, 17953 (1994).

25 P. Hohenberg and W. Kohn, Phys. Rev. 136, B864 (1964).

26 W. Kohn and L. J. Sham, Phys. Rev. 140, A1133 (1965).

27 G. Kresse and J. Hafner, Phys. Rev. B 47, 558 (1993).

28 G. Kresse and J. Furthmuller, Phys. Rev. B 54, 11169 (1996).

29 J. P. Perdew, K. Burke and M. Ernzerhof, Phys. Rev. Lett. 77, 3865 (1996).

30 H. J. Monkhorst and J. D. Pack, Phys. Rev. B 13, 5188 (1976).

31 P. Blaha, K. Schwarz, G. K. H. Madsen, D. Kvasnicka, and J. Luitz, WIEN2k, An Augmented Plane Wave + Local Orbitals Program for Calculating Crystal Properties, Karlheinz Schwarz Technische Universitat Wien, Austria, 2001, ISBN 3-9501031-1-2.

32 P. Giannozzi, S. Baroni, N. Bonini, M. Calandra, R. Car, C. Cavazzoni, D. Ceresoli, G. L. Chiarotti, M. Cococcioni and I. Dabo, J. Phys.: Condens. Matter 21, 395502 (2009).

33 G. Mills, H. Jonsson and G. K. Schenter, Surf. Sci. 324, 305 (1995).

34 F. Tran and P. Blaha, Phys. Rev. Lett. 102, 226401 (2009).

35 R. D. King-Smith and D. Vanderbilt, Phys. Rev. B 47, 1651 (1993).

36 V. I. Anisimov, J. Zaanen and O. K. Andersen, Phys. Rev. B 44, 943 (1991).

37 V. I. Anisimov, F. Aryasetiawan and A. Lichtenstein, J. Phys.: Condens. Matter 9, 767 (1997). 
38 J. Heyd, G. E. Scuseria and M. Ernzerhof, J. Chem. Phys. 118, 8207 (2003).

39 J. Heyd, J. E. Peralta, G. E. Scuseria and R. L. Martin, J. Chem. Phys. 123, 174101 (2005).

40 A. M. Glazer, Acta Crystallogr. B 28, 3384 (1972).

41 A. M. Glazer, Acta Crystallogr. A 31, 756 (1975).

42 P. M. Woodward, Acta Crystallogr. B 53, 44 (1997).

43 C. J. Howard and H. Stokes, Acta Crystallogr. B 54, 782 (1998).

44 C. J. Howard, B. J. Kennedy and P. M. Woodward, Acta Crystallogr. B 59, 463 (2003).

45 J. Li, J. Wang, M. Wuttig, R. Ramesh, N. Wang, B. Ruette, A. Pyatakov, A. Zvezdin and D. Viehland, Appl. Phys. Lett. 84, 5261 (2004).
${ }^{46}$ F. Bai, J. Wang, M. Wuttig, J. Li, N. Wang, A. Pyatakov, A. Zvezdin, L. Cross and D. Viehland, Appl. Phys. Lett. 86, 032511 (2005).

47 M. K. Singh, H. M. Jang, S. Ryu and M.-H. Jo, Appl. Phys. Lett. 88, 042907 (2006).

48 H. Bea, M. Bibes, X. Zhu, S. Fusil, K. Bouzehouane, S. Petit, J. Kreisel and A. Barthelemy, Appl. Phys. Lett. 93, 072901 (2008).

49 T. Kanai, S.-I. Ohkoshi and K. Hashimoto, J. Phys. Chem. Solids 64, 391 (2003).

50 S. J. Clark and J. Robertson, Appl. Phys. Lett. 90, 132903 (2007).

51 M. Lezaic and N. A. Spaldin, Phys. Rev. B 83, 024410 (2011). 